\title{
Modélisation écologique de la marée verte sur les côtes bretonnes
}

\author{
Ecological modelling of Ulva mass blooms on the Britanny coasts \\ THIERRY PERROT \\ CEVA, Service Ressources Naturelles et Environnement \\ Presqu'île de Pen-Lan - BP 3 - 22610 PLEUBIAN - France \\ Tel : +33 (0)2 962293 50, Fax : +33(0)2 962284 38, e-mail : thierry.perrot@ceva.fr
}

\author{
ALAIN MENESGUEN, FRANCK DUMAS \\ IFREMER, Département Dynamiques de l'Environnement Côtier \\ Technopôle Brest Iroise - BP 70 - 29280 PLOUZANE - France \\ Tel : +33 (0)2 98224334 - Fax : +33 (0)2 9845 48, e-mail : alain.menesguen@ifremer.fr
}

\begin{abstract}
$E$ very spring and summer, and for two decades, mass-blooms produced by a green alga of the genus Ulva, have been polluting some localities along the Brittany coasts. The main aspect of the nuisance lies in the stranding and further decomposition of large amounts of algae along recreational zones inducing visual and odoriferous pollution. Sites which are favourable to Ulva mass-blooms are shallow areas where water exchange with the open sea is slow (weak residual drift of water masses and long residence time). Drifting algae and nutrients are both trapped in these zones thus allowing Ulva thalli to be kept in good cultivation conditions. In collaboration with Ifremer, the socalled "MARS-ulve" ecological model (coupling the biochemical model ELISE with the hydrodynamical model MARS $2 D)$ has been developed. The main goal of the ecological model is to establish quantitative quality objectives relative to nitrate and ammonia in the tributaries of the main bays of Brittany severely polluted by Ulva "green tide ». A specific capability of this model is to allow quantifying the relative contribution of each source of inorganic supply in the feeding of the green tide. A new numerical technique which allows assessing the proportion of nitrogen in the nitrogen pool of the ulvae has been coupled with the ecological model and applied in the St Brieuc bay.
\end{abstract}

\section{I INTRODUCTION}

Cet article présente les résultats obtenus par le CEVA, en collaboration avec l'Ifremer/Brest, suite à la mise en place d'un modèle écologique "MARS-ulve » appliqué au développement des marées vertes sur le littoral breton. Ce modèle doit permettre en particulier de chiffrer la contribution relative des divers apports en sels nutritifs d'origine anthropique sur la prolifération algale du site étudié et d'évaluer l'impact de changements des apports en nutriments sur l'initiation et le développement de la marée verte. Le travail visant à la construction du modèle écologique se compose de deux parties successives et distinctes. La première partie concerne l'élaboration, le calage et la validation du modèle hydrodynamique bidimensionnel (MARS 2D) sur la zone de la baie de St Brieuc en Bretagne Nord. La seconde partie concerne, d'une part, le couplage du modèle biochimique «ELISE » appliqué aux ulves au modèle hydrodynamique MARS élaboré en baie de St Brieuc et, d'autre part, la calibration et la validation du modèle écologique sur ce site.

\section{DÉVELOPPEMENT - CALAGE ET VALIDATION DU MODÈLE HYDRODYNAMIQUE MARS}

\section{II.1 DÉFINITION DES EMPRISES RESPECTIVES DES EMBOÎTEMENTS DE MODÈLES 2D}

L'objectif du modèle hydrodynamique bidimensionnel développé dans en baie de St Brieuc est de fournir les champs de courants barotropes et de hauteur d'eau pour permettre le calcul des processus d'advection - dispersion des différentes variables d'état du modèle biogéochimique et leur évolution biologique. Les conditions aux limites du modèle hydrodynamique de ce modèle sont obtenues par la technique des modèles emboîtés, qui nécessite le développement de modèles 2D d'emprises décroissantes et de résolution croissante. L'utilisation systématique d'emboîtement de modèle 2D sur la zone de Bretagne nord est justifiée par la marée de type barotrope et l'absence de stratification thermique. Le premier modèle (appelé rang 0) a une résolution de $5 \mathrm{~km}$ et s'étend en latitude du milieu de l'Espagne au nord de la Norvège et en longitude de l'Islande à l'Italie $\left(39^{\circ} \mathrm{N}\right.$ $-65^{\circ} \mathrm{N}$ et $20^{\circ} \mathrm{W}-15^{\circ} \mathrm{E}$ ). Ce modèle est forcé aux frontières ouvertes à partir de huit constituants de marée diurnes et semi-diurnes issus de l'Atlas de Schwinderski [5]. Le second modèle (appelé rang 1) a une résolution de $900 \mathrm{~m}$ et fournit les conditions en hauteur d'eau à un troisième modèle intermédiaire (appelé rang 2) qui a une résolution de $500 \mathrm{~m}$. Ce modèle fournit à son tour les conditions aux limites au modèle de détail de la baie de St Brieuc (appelé rang 3) de résolution $150 \mathrm{~m}$. La figure 1 illustre l'emprise du modèle de rang 0 et des modèles de rang 1,2 et 3 .

\section{II.2 VALIDATION DU MODÈLE DE DÉTAIL DE LA BAIE DE ST BRIEUC}

Lévaluation de la qualité du modèle hydrodynamique de détail de la baie de St Brieuc (rang 3) pour la reproduction des niveaux d'eau a consisté en la comparaison en plusieurs points fixes, traduites en décalage temporel et en différence 

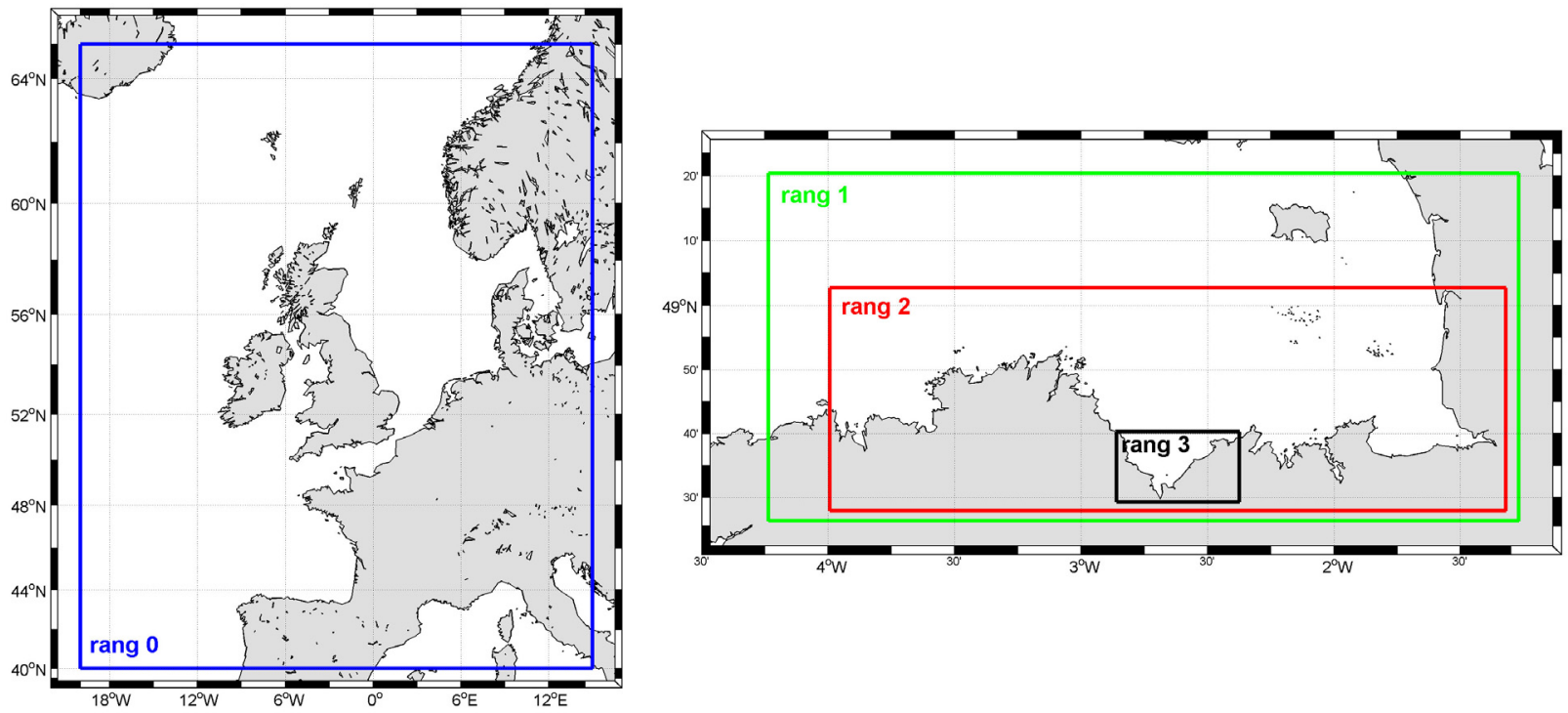

Figure 1 : Emprises des modèles de rang 0 (figure de gauche) et de rang 1, 2 et 3 (figure de droite).

de marnage par rapport au marnage moyen, des hauteurs d'eau calculées par le modèle avec celles prédites par les données du fichier CST_France du SHOM [2] qui fournit les amplitudes et les phases des 115 premiers constituants du signal de marée. Les données du fichier CST_France mêlent les résultats de simulations $2 \mathrm{D}$ (utilisation du modèle TELEMAC 2D) avec des résultats de mesures in-situ réalisées en Manche et sur le plateau continental du Golfe

Evolution des hauteurs d'eau (en $\mathrm{m}$ ) en un point fixe de la baie de St Brieuc (niveau moyen retiré)

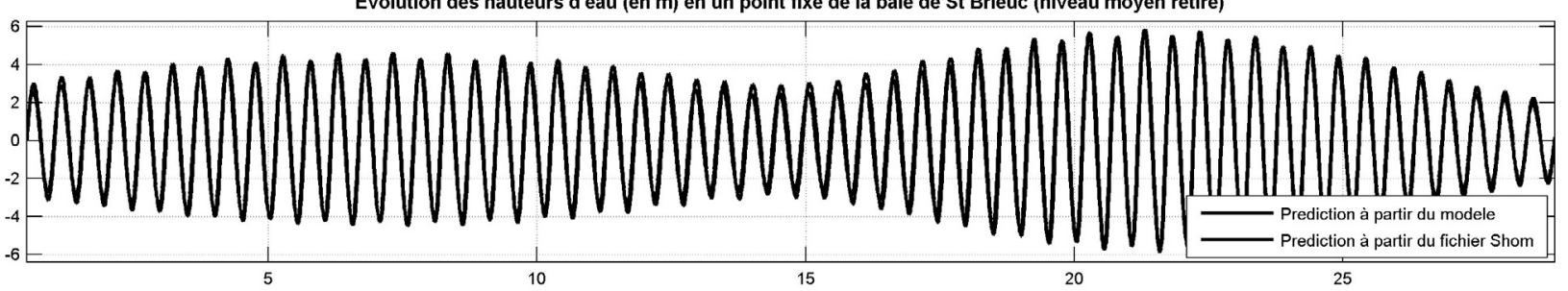

Evolution du decalage temporel (en mn) entre la prédiction Shom et le modèle - $\mid$ valeur moyenne $\mid=\mathbf{2 1 . 3 1 7 5} \mathbf{~ m n}$

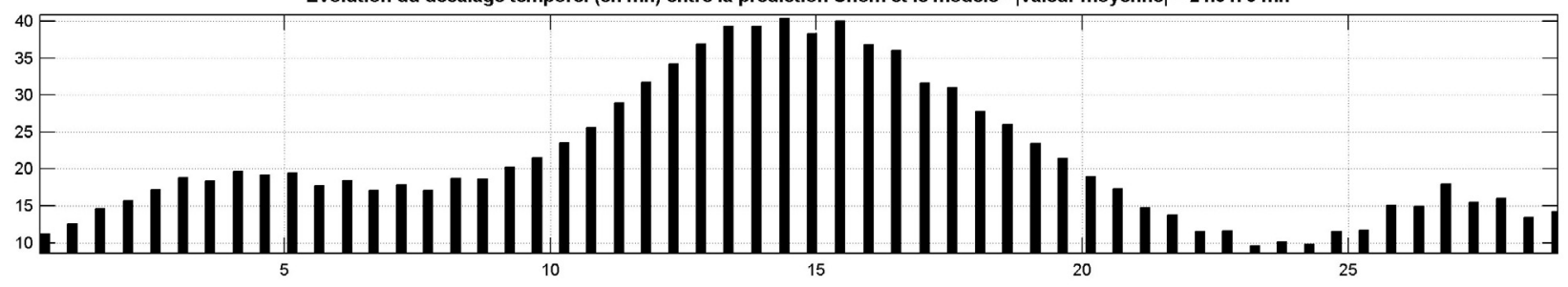

Evolution de l'erreur relative de marnage $(e n \%)$ entre la prédiction Shom et le modèle- |valeur moyenne $\mid=\mathbf{1 3 . 8 8 5 1} \%$

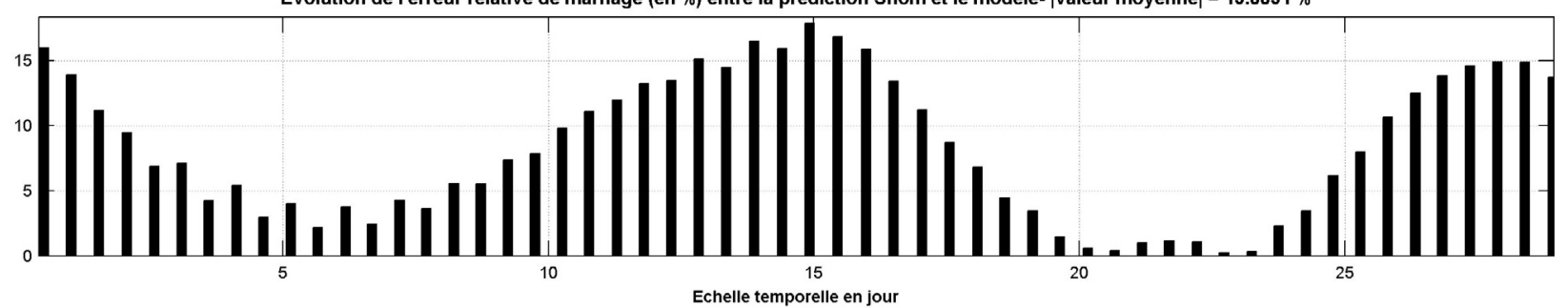

Figure 2 : Evolution du déphasage moyen et de l'erreur relative moyenne de marnage obtenus pour un point fixe de la baie de St Brieuc $\left(2.6774^{\circ} \mathrm{W}, 48.6571^{\circ} \mathrm{N}\right)$ entre la solution du modèle et la solution estimée à partir des constantes harmoniques du fichier CST_France (moyenne réalisée sur la prédiction du mois de janvier 1996). 
de Gascogne au moyen de marégraphes. Les résultats des simulations présentés dans ce rapport ont été obtenus en l'absence de vent et sans prise en compte des effets de la pression atmosphérique. Les comparaisons réalisées sur les hauteurs d'eau reposent sur la prédiction du mois de janvier 1996. La figure 2 présente, en un point fixe localisé au nord de la baie, les résultats de l'évolution du déphasage moyen et de la différence de marnage moyenne entre la solution prédite par le modèle de détail de la baie de St Brieuc et la solution estimée au moyen de la prédiction SHOM.

Les résultats présentés en figure 2 montrent une tendance marquée à l'augmentation (respectivement à la diminution) du déphasage et de la différence de marnage par rapport au marnage moyen en période de morte-eau (respectivement en période de vive-eau). L'erreur relative moyenne de marnage sur l'ensemble du mois de janvier 1996 entre la prédiction Shom et le modèle est de l'ordre de $13 \%$ tandis que le décalage temporel moyen est légèrement supérieur à 20 minutes.

La validation des courants de marée barotrope calculés par le modèle a consisté, au moyen des atlas de courants de marée du SHOM [6] édités sous forme de documents papier, à comparer sur un cycle de marée la direction et l'intensité des champs de courants fournis par le SHOM et le modèle. Globalement, on observe que les champs de courants calculés par le modèle sont cohérents avec les données SHOM (cf. figures 3 et 4) mais la comparaison est cependant rendue difficile étant donné le manque de données et leur qualité en terme de résolution spatiale et temporelle.

\section{COUPLAGE DU MODÈLE BIOCHIMIQUE AU MODĖLE HYDRODYNAMIQUE MARS ET CALIBRATION DU MODÉLE ECOLOGIQUE EN BAIE DE ST BRIEUC}

\section{III.1 CALIBRATION DU MODÈLE ÉCOLOGIQUE EN BAIE DE ST BRIEUC}

L'année de référence choisie pour les simulations est l'année 2001. Dans le but de simuler le cycle saisonnier de la production en ulve du site de St Brieuc, il est nécessaire de spécifier les données de forçage qui ont été collectées auprès de divers organismes: Météo France pour les données de température de l'air, de pression atmosphérique de vent, de nébulosité et d'humidité de l'air (données issues du modèle Arpège), Agence de l'Eau Loire-Bretagne pour les données de flux et de température des rivières et la banque HYDRO (Banque nationale de données pour l'hydrométrie et l'hydrologie) pour les données de débits des rivières du fond de baie de St Brieuc. La calibration du modèle écologique
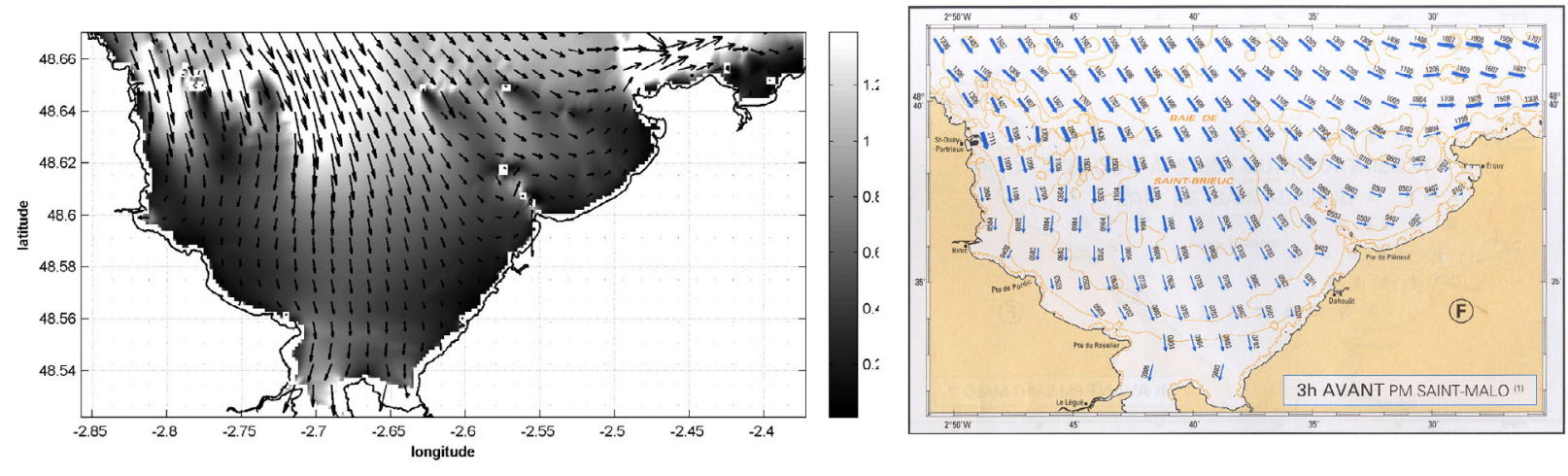

Figure 3 : Courants de marée issus du modèle de St Brieuc pour un coefficient de marée de 99 (figure de gauche) et de l'Atlas du SHOM (figure de droite) en vive-eau moyenne à BM + 3h (PM à St Malo).
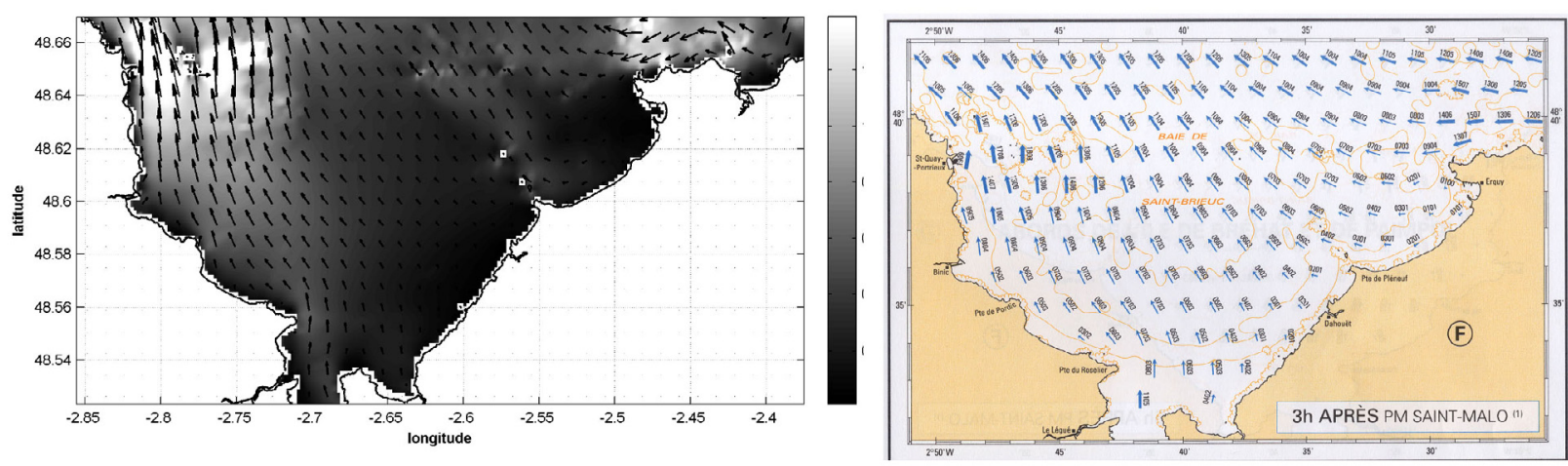

Figure 4 : Courants de marée issus du modèle de St Brieuc pour un coefficient de marée de 99 (figure de gauche) et de l'Atlas du SHOM (figure de droite) en vive-eau moyenne à PM + 3h (PM à St Malo). 


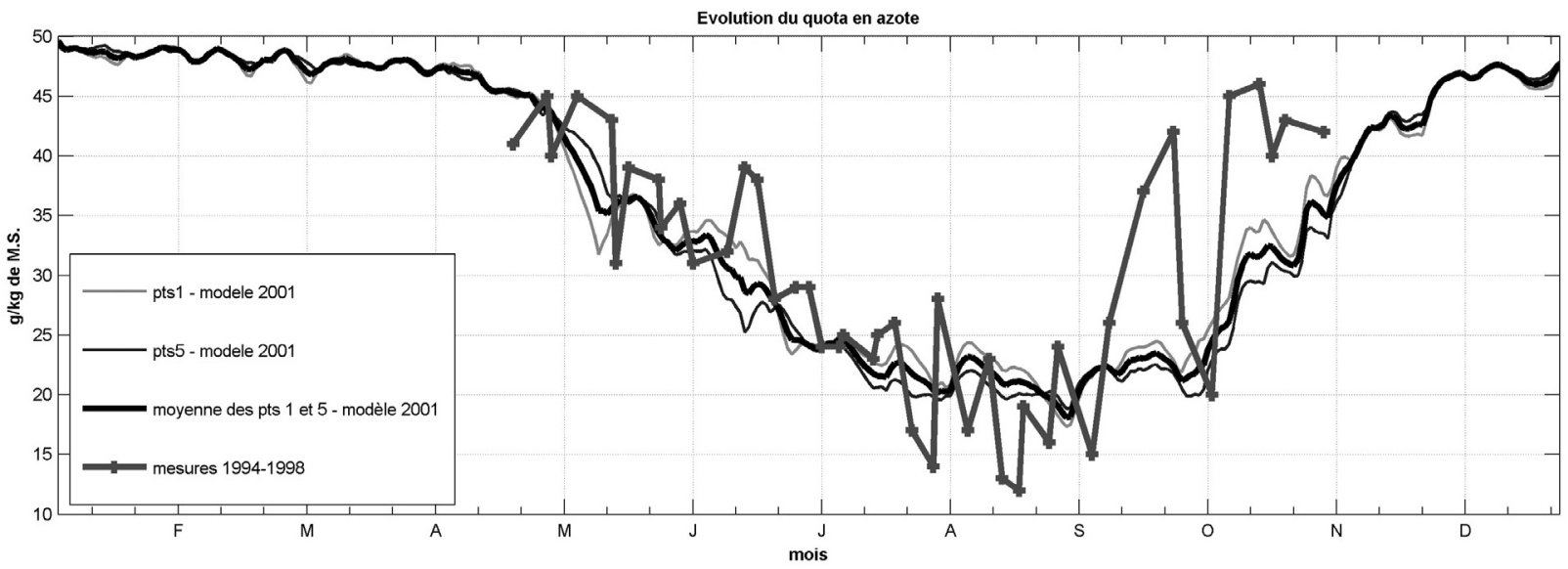

Figure 5 : Evolution calculée du quota en azote en 2 points fixes (pts1 : 2,6326 ${ }^{\circ} \mathrm{W}, 48,544^{\circ} \mathrm{N}-\mathrm{pts}^{2}: 2,651^{\circ} \mathrm{W}$, $48,5359^{\circ} \mathrm{N}$ ) et comparaison avec les mesures réalisées en 1998, 2002, 2003 et 2004 à Lermot.

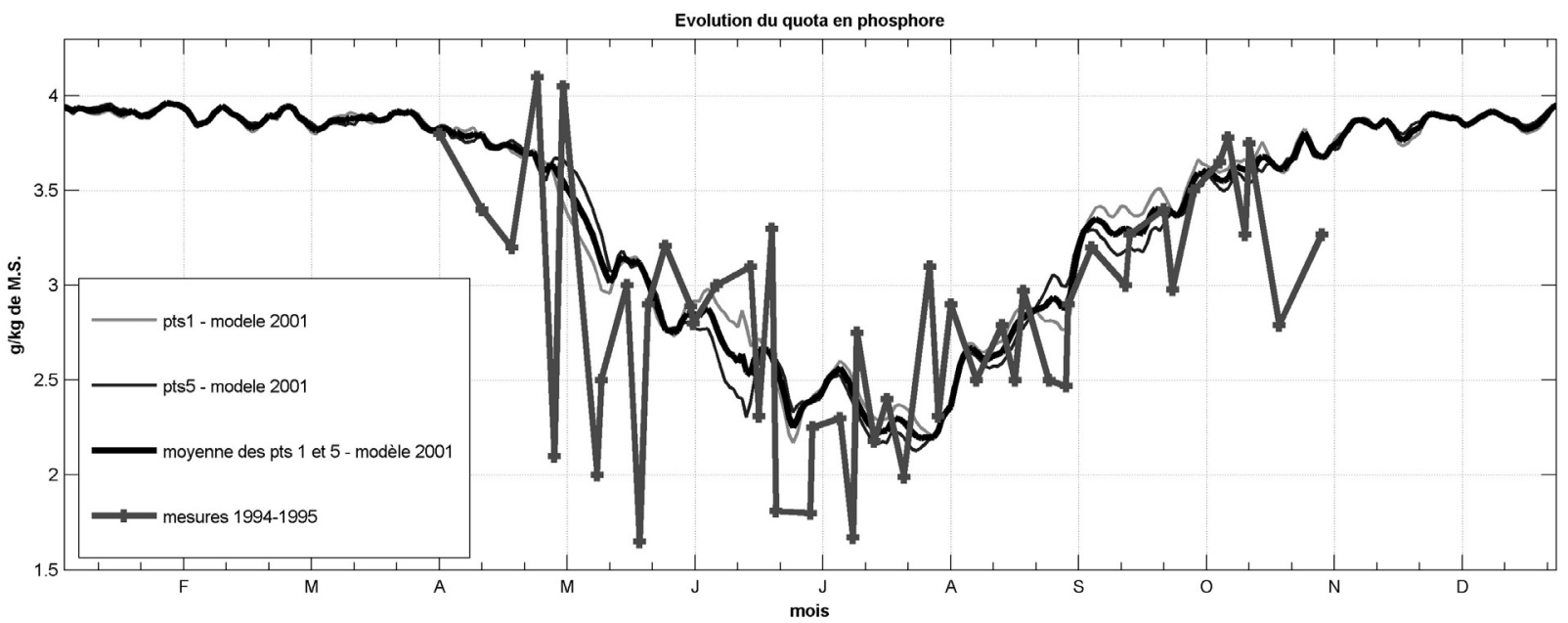

Figure 6 : Evolution calculée du quota en phosphore en 2 points fixes (pts1 : 2,6326 $\mathrm{W}, 48,544^{\circ} \mathrm{N}-\mathrm{pts}^{\circ}: 2,651^{\circ} \mathrm{W}$, $48,5359^{\circ} \mathrm{N}$ ) et comparaison avec les mesures réalisées en 1994 et 1995 à Gouessant.

a consisté d'abord à comparer l'évolution calculée par le modèle en 2001 pour les quotas internes des ulves en azote et en phosphore (cf. figures 5 et 6 ) avec des mesures ponctuelles pluri-annuelles. La calibration s'est poursuivie en comparant l'évolution de la biomasse prédite par le modèle avec des mesures ponctuelles concernant la biomasse totale (cf. figure 8) et la surface des dépôts sur estran. La figure 7 illustre la cartographie des dépôts au maximum estival de biomasse.

Concernant l'évolution des contenus internes en azote et en phosphore, on observe que le modèle reproduit correctement l'évolution du quota en azote et dans une moindre mesure celui en phosphore. La comparaison des mesures in situ effectuées par le Ceva avec le modèle est cependant rendue difficile par la forte variabilité pluri-annuelle des mesures disponibles et l'absence de mesures pour l'année 2001 simulée. Sur la période comprise entre début avril et fin août, l'analyse de l'évolution du quota en azote montre que la nutrition a été globalement limitée par rapport aux besoins de croissance de l'ulve tandis que le quota en phosphore demeure à un niveau élevé bien au-delà du quota critique de $1 \mathrm{~g} / \mathrm{kg}$ de M.S. L'azote apparaît donc comme le facteur limitant de la croissance des algues, alors que le phosphore est lui au contraire disponible dans le milieu au-delà des besoins de cette croissance (en raison notamment de sa capacité de stockage dans les sédiments littoraux).

La cartographie de la biomasse des ulves en dépôt montre que les ulves se confinent en certains lieux privilégiés. Il s'agit de la zone intertidale du fond de baie de St Brieuc (anses de Morieux et d'Yffiniac) qui s'avère être une zone de confinement hydrodynamique où la lumière et les sels nutritifs sont disponibles pour les besoins de croissance des algues. La courbe d'évolution de la biomasse totale d'ulves pour le fond de baie de St Brieuc rend compte du phénomène de bloom (pente de la courbe de croissance élevée à partir de mai) ainsi que de la décroissance plus lente et quasi 


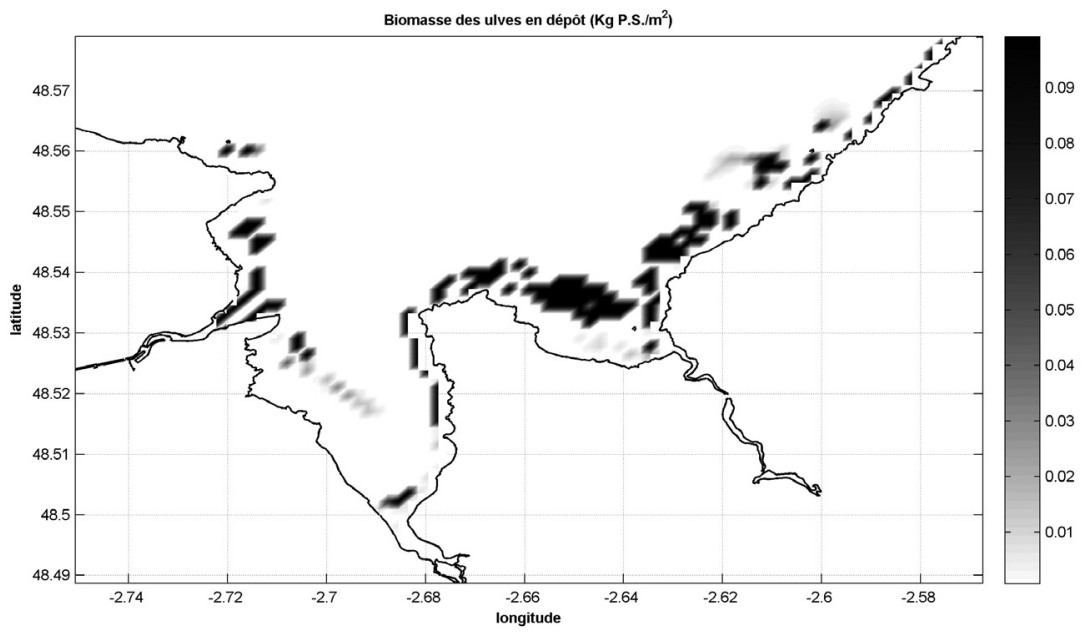

Figure 7 : Cartographie de la biomasse des ulves en dépôt (en kg de poids sec) du fond de baie de St Brieuc estimée par le modèle au 21 juillet 2001.

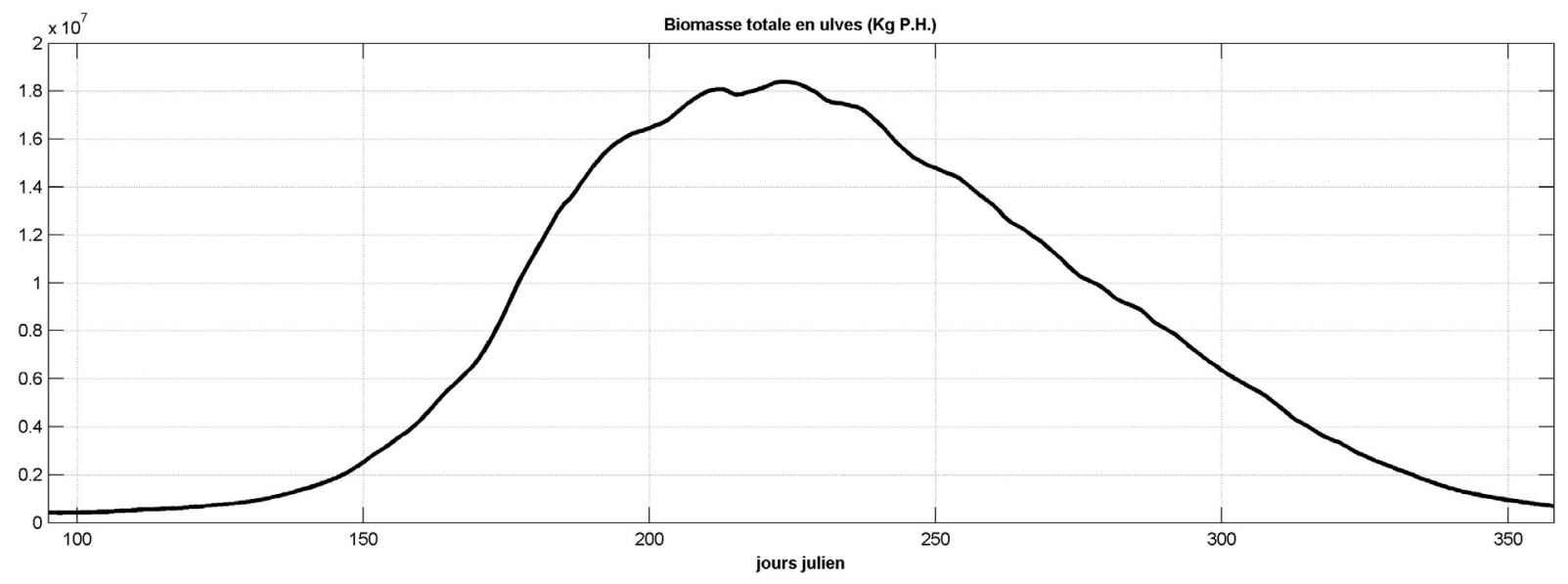

Figure 8 : Evolution de la biomasse totale en ulves (en kg de poids humide) du fond de baie de St Brieuc (anses de Morieux et Yffiniac) calculée par le modèle.

linéaire de la biomasse à partir de fin août. Bien que la comparaison soit difficile à réaliser du fait de la haute variabilité interannuelle du stock sur estran et des ramassages effectués en fond de baie de St Brieuc, l'ordre de grandeur du stock total obtenu par simulation (un peu plus de 18000 tonnes en poids humide) peut être considéré en accord avec les estimations de la biomasse sur estran réalisées par le Ceva où l'on relevait le 22 août 2002 une biomasse sur estran de l'ordre de 13000 tonnes contre 25000 tonnes le 06 juillet 2005 .

\section{III.2 EXPLOITATION DU MODÈLE ÉCOLOGIQUE EN BAIE DE ST BRIEUC}

III.2.1 Fraction de l'azote des ulves du fond de baie de St Brieuc issue des diverses sources d'azote d'origine terrestre et marine
Cette méthode innovante décrite dans [4] permet de quantifier et de suivre sur l'ensemble de la simulation la contribution relative de chaque source d'azote à l'alimentation de la marée verte se développant sur le site étudié. La méthode consiste à simuler en parallèle du cycle nominal le cycle d'un traceur issu d'une source particulière. Pour cela, on réalise pour chaque variable d'état transportée participant au cycle de l'azote une copie de son équation d'advection-diffusion-réaction en faisant subir non plus à la variable d'état seule mais à la "variable d'état*fraction d'azote issue d'une source d'azote particulière » les mêmes processus d'advection, dispersion, création-disparition biochimiques. Cette approche, relativement lourde sur le plan numérique, présente l'énorme avantage de ne rien perturber de la simulation nominale contrairement à la suppression d'une des sources d'azote. Les résultats sont présentés en figure 9 . 


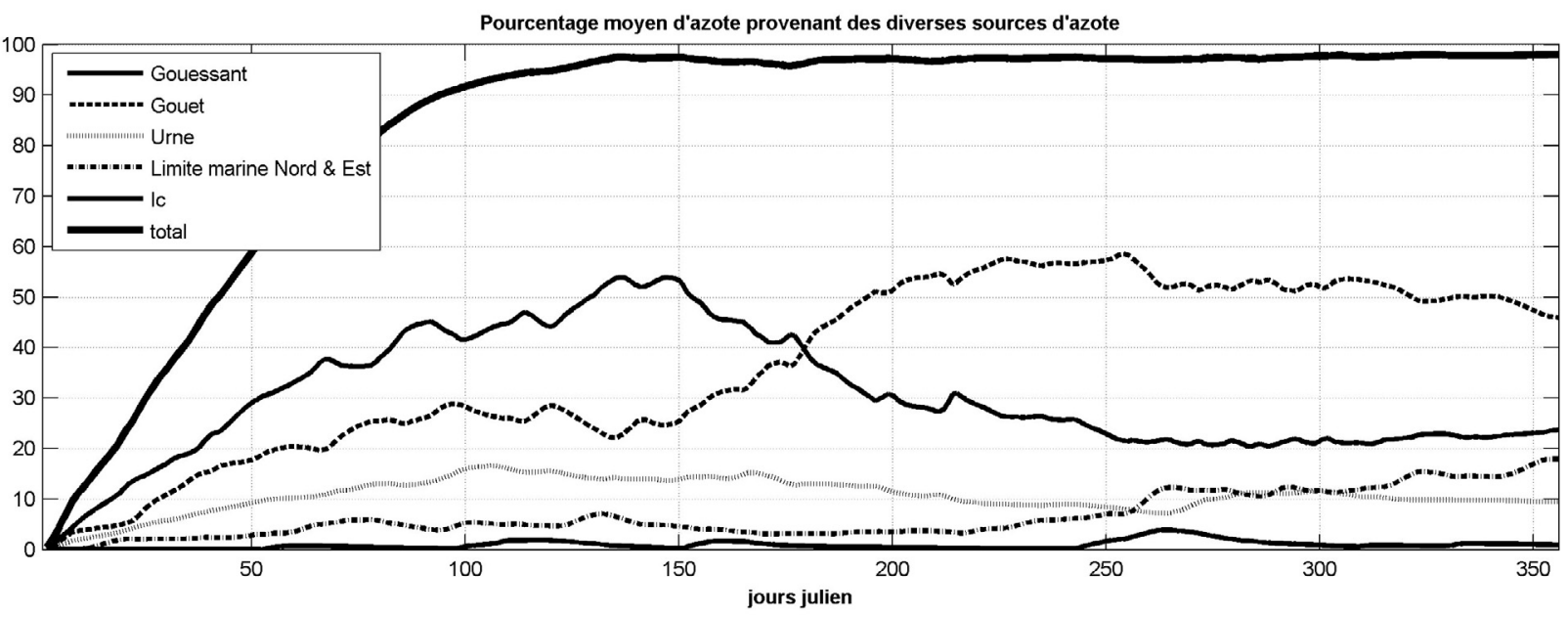

Figure 9 : Origine calculée de l'azote contenue dans les ulves du fond de baie de St Brieuc en situation nominale.

Tableau 1. Résultats des scenarii de réduction des apports azotés par rapport à la situation réelle de 2001 au moyen du modèle MARS-ulve et de 1994 au moyen du modèle en boîtes ELISE.

\begin{tabular}{|c|c|c|}
\hline Situation & $\begin{array}{c}\text { \% abattement par rapport } \\
\text { à la situation réelle de 2001 } \\
\text { pour le fond de baie de St Brieuc }\end{array}$ & $\begin{array}{c}\text { \% abattement par rapport } \\
\text { à la situation réelle de 1994 } \\
\text { pour le fond de baie de St Brieuc }\end{array}$ \\
\hline Sans aucun apport terrestre de NO3 et NH4 & $\mathbf{9 6}$ & $\mathbf{9 1}$ \\
\hline Sans apport NO3 et NH4 par Gouët & $\mathbf{3 7}$ & $\mathbf{8}^{*}$ \\
\hline Sans apport NO3 et NH4 par Gouessant & $\mathbf{1 6}$ & $\mathbf{1 1}^{*}$ \\
\hline Sans apport NO3 et NH4 par Urne & $\mathbf{3}$ & $\mathbf{3}^{*}$ \\
\hline Sans apport NO3 et NH4 par Ic & 0.2 & - \\
\hline $\begin{array}{c}\text { Sans apport NO3 et NH4 par les Limites } \\
\text { marines Nord et Est }\end{array}$ & $\mathbf{2 , 2}$ & - \\
\hline
\end{tabular}

* Sans apport de NO3 uniquement.

\section{III.2.2 Rôle respectifs des divers apports en azote sur la marée verte du fond de baie de St Brieuc}

Un seul type de scénario a été testé afin de montrer la capacité opérationnelle du modèle écologique à répondre à une modification quantitative des apports azotés issus des différentes sources d'azote (apports terrigènes par les rivières et aux limites marines). En gardant l'année climatique 2001, le "scénario descendant» testé a consisté à supprimer totalement les apports de nitrate et ammonium pour chaque source d'azote prise à tour de rôle et à évaluer l'impact sur la production annuelle de biomasse. Le tableau 1 synthétise les résultats obtenus en termes de pourcentage d'abattement par rapport à la situation réelle de 2001, comparés à ceux obtenus précédemment par rapport à la situation de 1994 au moyen du modèle en boîtes ELISE dans le cadre de l'étude [3].

\section{CONCLUSIONS}

Les premiers scénarios de réduction des apports azotés obtenus à l'issue de l'exploitation du modèle écologique
MARS-ulve élaboré en baie de St Brieuc permettent de tirer les conclusions suivantes:

- Ce sont bien les apports d'azote inorganique dissous $(\mathrm{NO} 3+\mathrm{NH} 4)$ par les rivières qui sont responsables de la production excessive d'ulves sur les estrans du fond de baie de St Brieuc puisque la suppression de tous les apports d'origine terrestre réduit la marée verte de $96 \%$,

- Les apports d'azote dissous du Gouët ont un rôle sensiblement plus important que ceux du Gouessant (37\% contre $16 \%$ ). Ce résultat trouve son explication d'une part dans les fortes teneurs en ammonium rejetées par le Gouët en période estivale et d'autre part dans le fait que le panache de dispersion issue du Gouët alimente fréquemment l'anse voisine de Morieux contribuant ainsi de manière optimale à l'alimentation de la zone intertidale du fond de baie de St Brieuc en azote dissous,

- Une suppression totale des apports azotés du Gouët seul ou du Gouessant seul n'est pas en mesure de réduire sensiblement la marée verte,

- Les apports issus de l'Urne contribuent très peu à l'alimentation de la marée verte du fond de baie de St Brieuc $(3 \%)$, tandis que la rivière Ic joue un rôle négligeable, 
- Les apports azotés d'origine marine contribuent également peu à la marée verte du fond de baie de St Brieuc.

Malgré la complexité hydrodynamique du site mal prise en compte par le modèle en boîtes ELISE (transport advectif et/ou dispersif entre les boîtes estimé à partir du champ de courants résiduels de marée calculé par [1]), les résultats des scénarios de réduction des apports azotés estimés par le modèle en boîtes ELISE et par le modèle MARS-ulve montrent des tendances proches. L'écart significatif observé entre les deux modèles précisément pour la rivière du Gouët est dû au fait que le scénario testé par le modèle en boîte ne prenait pas en compte la suppression des rejets d'ammonium particulièrement élevés sur ce cours d'eau en raison de la présence d'une station d'épuration.

D'autre part, l'évolution calculée et moyennée entre les jours juliens 200 et 350 du pourcentage d'azote des ulves $\mathrm{du}$ fond de baie de St Brieuc issus des diverses sources d'azote (Gouët, Gouessant, Urne, Ic, limites marines Nord et Est) confirme bien le rôle important du Gouët puisque cette source parvient à constituer un peu plus de $50 \%$ de l'azote contenu dans les ulves contre $25 \%$ pour le Gouessant, $9 \%$ pour l'Urne, $11 \%$ pour les apports marins et une quantité négligeable pour l'Ic (moins de $2 \%$ ).

\section{DÉFÉRENCES ET CITATIONS}

[1] Garreau P. (1993). — Conditions hydrodynamiques sur la côte nord-Bretagne. Programme d'étude. Cartographie des zones sensibles à l'eutrophisation. Rapport IFERMER DEL/93.02.20 p. +annexes.
[2] Le Roy R. EtSimon B. (2003). — Réalisation et validation d'un modèle de marée en Manche et dans le Golfe de Gascogne. Application à la réalisation d'un nouveau programme de réduction des sondages bathymétriques. Rapport technique, EPSHOM. 002/03, 2003.

[3] MÉnesguen A. (1998). - Détermination d'objectifs de qualité en nutriments dissous pour les rivières alimentant la marée verte des baies de Saint-Brieuc et Lannion. Rapport final de contrat pour le Conseil Général des Côtes d'Armor, 24 p.

[4] Ménesguen A., Cugier P., Leblond I. (2006). - A new numerical technique for tracking chemical species in a multisource, coastal ecosystem applied to nitrogen causing Ulva blooms in the Bay of Brest (France) Limnol. Oceanogr. 51(1, part 2) 591-601.

[5] Schwiderski E.W. (1978). - Global ocean tides, part I : a detailed hydrodynamical interpolation model. Naval Surface Weapons Centre Technical Report, 3866.

[6] Sном G. (2000). - Courant de marée et hauteurs d'eau. La Manche, de Dunkerque à Brest. - Rapport technique, SHOM. 